\title{
Lower limit of the transverse emittance growth in deflecting rf fields
}

\author{
V. Paramonov $\oplus^{*}$ \\ Institute for Nuclear Research of the RAS, 117312, Moscow, Russia \\ K. Floettmann \\ Deutsches Elektronen-Synchrotron, Notkestraße 85, 22607 Hamburg, Germany
}

(Received 12 December 2018; published 9 January 2020)

\begin{abstract}
The Panofsky-Wenzel theorem connects the transverse deflecting force in an rf structure with the existence of a longitudinal electric field component. In this paper it is shown that a transverse deflecting force is accompanied by an additional longitudinal magnetic field component which leads to an emittance growth in the direction perpendicular to the transverse force. The general description of transverse deflecting waves requires a linear combination of basis modes to cope with the boundary conditions and to support the simultaneous existence of electric and magnetic longitudinal field components. The mode description is preferably performed in the hybrid HM-HE basis to avoid convergence problems, which are fundamental for the transverse TM-TE basis.
\end{abstract}

DOI: $10.1103 /$ PhysRevAccelBeams.23.014401

\section{INTRODUCTION}

Transverse deflecting rf structures find numerous applications in modern particle accelerators for example as particle separators [1] or fast rf deflectors [2], as a streaking device for diagnostics purposes [3], in emittance exchange beam lines [4] or as crab cavities in circular colliders as the LHC [5]. It is well known, that a beam passing through a transverse deflecting field will not only receive the desired phase dependent transverse momentum, but it will also change its energy spread due to a longitudinal electric field component which varies over the transverse size of the beam. The fundamental relation of the transverse gradient of the longitudinal electric field component and the transverse momentum was formulated by Panofsky and Wenzel in their seminal paper in 1956 [6]. Originally derived in the context of transverse deflecting rf structures, which is also the focus of this paper, the PanofskyWenzel theorem became a fundamental relation also for the discussion of wake potentials [7] and devices as pickups and kickers [8].

Complementary to the longitudinal electric field component a longitudinal magnetic field component exists in transverse deflecting rf fields which has been widely ignored so far. Due to the coupling of the transverse motion to the longitudinal magnetic field it leads to a small, but

\footnotetext{
"paramono@inr.ru
}

Published by the American Physical Society under the terms of the Creative Commons Attribution 4.0 International license. Further distribution of this work must maintain attribution to the author(s) and the published article's title, journal citation, and DOI. fundamental contribution to the transverse beam emittance in the direction perpendicular to the transverse force.

\section{GENERAL RELATIONS}

The transverse deflecting force $\vec{F}_{\perp}$ acting on a particle moving along the longitudinal axis $z$ with the velocity $\vec{V}=\vec{i}_{z} v_{z}=\vec{i}_{z} \beta_{z} c$ is defined by the transverse components of the Lorentz force

$$
\begin{aligned}
& \vec{F}_{\perp}=e\left(E_{x}-\beta_{z} c B_{y}\right) \vec{i}_{x}+e\left(E_{y}+\beta_{z} c B_{x}\right) \vec{i}_{y}, \\
& \vec{F}_{\perp}=e\left(E_{r}-\beta_{z} c B_{\vartheta}\right) \vec{i}_{r}+e\left(E_{\vartheta}+\beta_{z} c B_{r}\right) \vec{i}_{\vartheta},
\end{aligned}
$$

where $\vec{i}_{x}, \vec{i}_{y}, \vec{i}_{z}$ and $\vec{i}_{\vartheta}, \vec{i}_{r}, \vec{i}_{z}$ are the basis vectors of the Cartesian and cylindrical coordinate system, respectively.

The fundamental relations of the deflecting and the corresponding longitudinal electric and magnetic field components follow directly from Maxwell's equations $\operatorname{curl} \vec{E}=-\frac{\partial}{\partial t} \vec{B}$ and $\operatorname{curl} \vec{B}=\frac{\partial}{\partial t} \frac{\vec{E}}{c^{2}}$ as:

$$
\begin{aligned}
\frac{\partial}{\partial x} E_{z} & =\frac{\partial}{\partial z} E_{x}+\frac{\partial}{\partial t} B_{y}, \\
\frac{\partial}{\partial y} c B_{z} & =\frac{\partial}{\partial t} \frac{E_{x}}{c}+\frac{\partial}{\partial z} c B_{y} .
\end{aligned}
$$

For a wave oscillating with frequency $\omega$, phase velocity $v_{\mathrm{ph}}$ and wave number $k_{z}=\omega / v_{\mathrm{ph}}$ as $\propto \mathrm{e}^{i\left(\omega t-k_{z} z\right)}$ the relation $\frac{1}{\partial t}=-\frac{v_{\mathrm{ph}}}{\partial z}$ holds, thus 


$$
\begin{aligned}
\frac{\partial}{\partial x} E_{z} & =\frac{\partial}{\partial z}\left(E_{x}-v_{\mathrm{ph}} B_{y}\right), \\
-\frac{\partial}{\partial y} c B_{z} & =\frac{\partial}{\partial z}\left(\frac{v_{\mathrm{ph}}}{c} E_{x}-c B_{y}\right) .
\end{aligned}
$$

Equivalent transformations can be applied to the $E_{y}$ and $B_{x}$ components, resulting in

$$
\begin{aligned}
\frac{\partial}{\partial y} E_{z} & =\frac{\partial}{\partial z}\left(E_{y}+v_{\mathrm{ph}} B_{x}\right), \\
\frac{\partial}{\partial x} c B_{z} & =\frac{\partial}{\partial z}\left(\frac{v_{\mathrm{ph}}}{c} E_{y}+c B_{x}\right) .
\end{aligned}
$$

For a particle, traveling with longitudinal velocity component $v_{z}=v_{\mathrm{ph}}$ synchronously with the wave, the first equations in Eqs. (3) and (4) mean:

$$
\frac{1}{e} \frac{\partial}{\partial z} \vec{F}_{\perp}=-\frac{1}{e v_{\mathrm{ph}}} \frac{\partial}{\partial t} \vec{F}_{\perp}=-i \frac{k_{z}}{e} \vec{F}_{\perp}=\vec{\nabla}_{\perp} E_{z}
$$

where $\vec{\nabla}_{\perp}=\vec{i}_{x} \frac{\partial}{\partial x}+\vec{i}_{y} \frac{\partial}{\partial y}$, see [9] and references therein.

A synchronous transverse force $\vec{F}_{t} \neq 0$ can thus only exist together with a longitudinal component of the electric field $E_{z}$ in a deflecting rf field.

The second equations in Eqs. (3) and (4) suggest the simultaneous existence of a longitudinal magnetic field component and for the case $v_{z}=v_{\mathrm{ph}}=c$ the relation

$$
c\left[\vec{i}_{z} \times \vec{\nabla} B_{z}\right]_{\perp}=-i \frac{k_{z}}{e} \vec{F}_{\perp}=\vec{\nabla}_{\perp} E_{z}
$$

holds. This relation is a necessary and sufficient condition for existence of a wave with $\vec{F}_{\perp} \neq 0$ at $v_{\text {ph }}=c$, [9]. For a structure, supporting the wave with $\vec{F}_{\perp} \neq 0$ at $v_{\mathrm{ph}}=c$, from continuity of field components for $v_{\mathrm{ph}} \rightarrow c$ follows $\vec{F}_{\perp} \neq 0$ and existence of longitudinal component $B_{z} \neq 0$ at least in the vicinity $v_{\mathrm{ph}} \approx c$.

In deflecting structure for ultrarelativistic and relativistic particles a transverse force $\vec{F}_{\perp}$ is thus accompanied by both, a longitudinal electric, as well as a longitudinal magnetic field component.

\section{SUPPORTING STRUCTURE AND FIELD DESCRIPTION}

To achieve a synchronous interaction of an rf wave and a particle moving with $\beta_{z} \leq 1.0$, it is necessary to slow down the wave in an appropriate supporting structure.

Commonly periodically iris loaded structures as the example shown in Fig. 1, left, are employed for this purpose. The field distribution in a periodical structure represents a sum of spatial harmonics and by proper selection of the period length $d=\frac{\beta_{z} \lambda \theta}{2 \pi}, 0 \leq \theta \leq \pi$ a synchronous harmonic can be generated. $\theta$ is the phase advance per period and the wavelength $\lambda$ for this kind of structures is typically in the range of $20-3 \mathrm{~cm}$ (L-to $\mathrm{X}$-band). A fundamental property of periodical structures is the appearance of spatial harmonics which lead to nonlinearities in the field distribution. The nonlinearities can not be completely eliminated but they can be minimized in the region occupied by the beam by a proper design of the structure $[10,11]$.

Spatial harmonics do not appear in structures which are uniform in the longitudinal $z$ direction. To slow down the wave the structure can be partially filled with a dielectric medium (see Fig. 1, right) but also thin metallic layers [12] or even a rough surface [13] can lead to slow waves.

Dielectric lined waveguides have been discussed already in the 1960s [14,15] as candidates for beam separators operating at typical $\mathrm{rf}$ frequencies $(3 \mathrm{GHz})$. They gain now interest again as streaking device for diagnostics purposes operating in the sub- $\mathrm{THz}$ to $\mathrm{THz}$ range [16], where the dimensions are so small that the production of periodical structures reaches technical limits. The demand to resolve ever shorter bunch length and also the progress in the generation of $\mathrm{THz}$ pulses of sufficient power and pulse length [17] are driving forces of these developments. Moreover, the absence of spatial harmonics makes dielectric lined waveguides also attractive from the beam dynamics point of view, because side effects, like undesired emittance contributions, are minimized to their fundamental limits.

A general cylindrical symmetric representation of the electromagnetic fields in vacuum in a finite domain including the symmetry axis can be based on Hertzian basis vectors, see Ref. [18] and references therein for a general discussion. Table I summarizes the transverse TM-TE and the hybrid HM-HE basis vectors, as introduced in [18], with a modified notation (e.g., $\vec{H}$ has been replaced by $\vec{B}$ ). Here $k_{0}=\omega / c, k_{z}=\omega / v_{\mathrm{ph}}$ and $k_{r}^{2}=$ $k_{0}^{2}-k_{z}^{2}$ is used, while $n \in \mathbb{N}$ defines the azimuthal dependence.

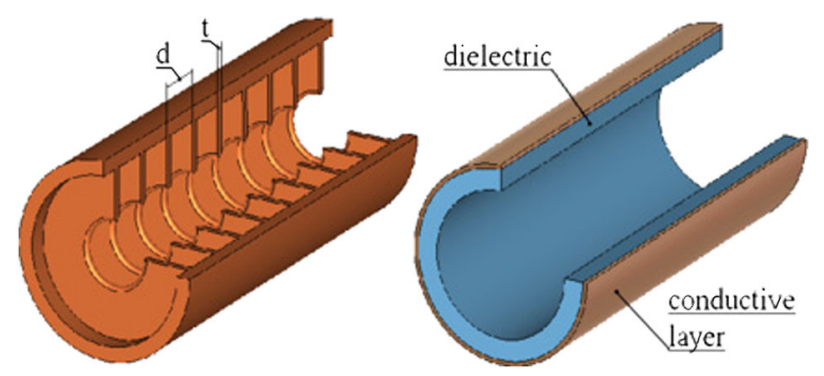

FIG. 1. Supporting structures for slow deflecting waves: periodical iris loaded circular waveguide, left, and dielectric lined waveguide, right. 
TABLE I. TM-TE and HM-HE basis vectors $\left(J_{n}=J_{n}\left(r k_{r}\right), J_{n}^{\prime}=\frac{\partial J_{n}\left(k_{r} r\right)}{\partial\left(k_{r} r\right)}=\frac{n J_{n}\left(k_{r} r\right)}{k_{r} r}-J_{n+1}\left(k_{r} r\right)\right)$.

\begin{tabular}{lcccccc}
\hline \hline & $\mathrm{TM}$ & $\mathrm{TE}$ & $\mathrm{HM}$ & $\mathrm{HE}$ & Azimuthal dependence & Spatio-temporal dependence \\
\hline$E_{r}$ & $-k_{z} \frac{J_{n}^{\prime}}{k_{r}^{n-1}}$ & $-\frac{n k_{0}}{r} \frac{J_{n}}{k_{r}^{n}}$ & $k_{0} k_{z} \frac{J_{n+1}}{k_{r}^{n+1}}$ & $k_{z}^{2} \frac{J_{n+1}}{k_{r}^{n+1}}+\frac{n}{r} \frac{J_{n}}{k_{r}^{n}}$ & $\cos (-n \vartheta)$ & $i \mathrm{e}^{i\left(\omega t-k_{z} z\right)}$ \\
$E_{\vartheta}$ & $\frac{n k_{z}}{r} \frac{J_{n}}{k_{r}^{n}}$ & $k_{0} \frac{J_{n}^{\prime}}{k_{r}^{n-1}}$ & $k_{0} k_{z} \frac{J_{n+1}}{k_{r}^{n+1}}$ & $k_{0}^{2} \frac{J_{n+1}}{k_{r}^{n+1}}-\frac{n}{r} \frac{J_{n}}{k_{r}^{n}}$ & $\sin (n \vartheta)$ & $i \mathrm{e}^{i\left(\omega t-k_{z} z\right)}$ \\
$E_{z}$ & $k_{r}^{2} \frac{J_{n}}{k_{r}^{n}}$ & 0 & $k_{0} \frac{J_{n}}{k_{r}^{n}}$ & $k_{z} \frac{J_{n}}{k_{r}^{n}}$ & $\cos (-n \vartheta)$ & $\mathrm{e}^{i\left(\omega t-k_{z} z\right)}$ \\
$c B_{r}$ & $-\frac{n k_{0}}{r} \frac{J_{n}}{k_{r}^{n}}$ & $-k_{z} \frac{J_{n}^{\prime}}{k_{r}^{n-1}}$ & $-k_{z}^{2} \frac{J_{n+1}}{k_{r}^{n+1}}-\frac{n}{r} \frac{J_{n}}{k_{r}^{n}}$ & $-k_{0} k_{z} \frac{J_{n+1}}{k_{r}^{n+1}}$ & $\sin (n \vartheta)$ & $i \mathrm{e}^{i\left(\omega t-k_{z} z\right)}$ \\
$c B_{\vartheta}$ & $-k_{0} \frac{J_{n}^{\prime}}{k_{r}^{n-1}}$ & $-\frac{n k_{z}}{r} \frac{J_{n}}{k_{r}^{n}}$ & $k_{0}^{2} \frac{J_{n+1}}{k_{r}^{n+1}}-\frac{n}{r} \frac{J_{n}}{k_{r}^{n}}$ & $k_{0} k_{z} \frac{J_{n+1}}{k_{r}^{n+1}}$ & $\cos (-n \vartheta)$ & $i \mathrm{e}^{i\left(\omega t-k_{z} z\right)}$ \\
$c B_{z}$ & 0 & $k_{r}^{2} \frac{J_{n}}{k_{r}^{n}}$ & $-k_{z} \frac{J_{n}}{k_{r}^{n}}$ & $-k_{0} \frac{J_{n}}{k_{r}^{n}}$ & $\sin (n \vartheta)$ & $\mathrm{e}^{i\left(\omega t-k_{z} z\right)}$ \\
\hline \hline
\end{tabular}

In the domain under consideration any field component $G$ can be described by a linear combination of the basis vectors as:

$G=A \times T M+B \times T E \quad$ or $\quad G=P \times H M+Q \times H E$

The coefficients $A, B, P$, and $Q$ are determined by the boundary conditions and the energy balance of the problem under consideration. The general solution has thus six field components and requires the linear combination of two basis vectors.

The TM-TE basis exhibits a methodical convergence problem when the phase velocity approaches $c$, because the longitudinal field components vanish as $k_{r}^{2}$. Comparing the longitudinal field components by means of Eq. (7) yields the following relations of the coefficients of the two basis:

$$
A=\frac{P k_{0}+Q k_{z}}{k_{r}^{2}}, \quad B=-\frac{P k_{z}+Q k_{0}}{k_{r}^{2}} .
$$

$A$ and $B$ are thus divergent $\propto k_{r}^{-2}$ when $k_{r}$ approaches zero. Thus, while the basis vectors converge to zero, the product of basis vectors with the vector coefficients does not converge to zero. The HM-HE basis of hybrid waves has no convergence problem. A detailed discussion of the representation of a deflecting field and the connection between TM-TE and HM-HE basis is presented in [19].

The expressions of the basis vectors in Table I are presented for $v_{\mathrm{ph}}>c$ and $k_{r}^{2}>0$. For waves with $v_{\mathrm{ph}}<c$, $k_{r}$ becomes imaginary and the modified Bessel functions should be used to describe the radial dependencies of the field components. For the relativistic case $v_{\mathrm{ph}}=c, k_{0}=k_{z}$, the relation

$$
\lim _{k_{r} \rightarrow 0} \frac{J_{n}\left(k_{r} r\right)}{k_{r}^{n}}=\frac{r^{n}}{2^{n} n !},
$$

holds. The field distribution of the synchronous deflecting wave, $n=1$, in the region of the interaction with the beam reads thus as:

$$
\begin{aligned}
E_{z} & =[P+Q] \frac{k_{0} r}{2} \cos (\vartheta) \mathrm{e}^{i \varphi}, \\
E_{r} & =i\left[P \frac{k_{0}^{2} r^{2}}{8}+Q\left(\frac{k_{0}^{2} r^{2}}{8}+\frac{1}{2}\right)\right] \cos (\vartheta) \mathrm{e}^{i \varphi}, \\
E_{\vartheta} & =i\left[P \frac{k_{0}^{2} r^{2}}{8}+Q\left(\frac{k_{0}^{2} r^{2}}{8}-\frac{1}{2}\right)\right] \sin (\vartheta) \mathrm{e}^{i \varphi}, \\
c B_{z} & =-[P+Q] \frac{k_{0} r}{2} \sin (\vartheta) \mathrm{e}^{i \varphi}, \\
c B_{r} & =-i\left[P\left(\frac{k_{0}^{2} r^{2}}{8}+\frac{1}{2}\right)+Q \frac{k_{0}^{2} r^{2}}{8}\right] \sin (\vartheta) \mathrm{e}^{i \varphi}, \\
c B_{\vartheta} & =i\left[P\left(\frac{k_{0}^{2} r^{2}}{8}-\frac{1}{2}\right)+Q \frac{k_{0}^{2} r^{2}}{8}\right] \cos (\vartheta) \mathrm{e}^{i \varphi},
\end{aligned}
$$

where $\varphi=\omega t-k_{0} z$ is the phase of the wave with respect to the particle.

Equation (10) is a general representation of the deflecting field, which does not depend on the supporting structure. It describes, for example, the field in a dielectric lined waveguide [14]. One can also show that the wellknown expressions for the field distribution in periodically iris loaded structures, (Fig. 1) derived in the so-called small pitch approximation (cf. [9]), are a particular case of the more general relations in Eq. (10) [19].

The transverse force, Eq. (1), follows for $\beta_{z}=1$ from Eq. (10) as:

$$
\begin{aligned}
& F_{r}=i e \frac{P+Q}{2} \cos (\vartheta) \mathrm{e}^{i \varphi}=i e \frac{\hat{E}}{2} \cos (\vartheta) \mathrm{e}^{i \varphi}, \\
& F_{\vartheta}=-i e \frac{P+Q}{2} \sin (\vartheta) \mathrm{e}^{i \varphi}=-i e \frac{\hat{E}}{2} \sin (\vartheta) \mathrm{e}^{i \varphi},
\end{aligned}
$$

or, transferring to Cartesian coordinates, as:

$$
\begin{array}{ll}
F_{x}=i e \frac{\hat{E}}{2} \mathrm{e}^{i \varphi}, & F_{y}=0, \\
E_{z}=\frac{\hat{E}}{2} k_{0} x \mathrm{e}^{i \varphi}, & c B_{z}=-\frac{\hat{E}}{2} k_{0} y \mathrm{e}^{i \varphi} .
\end{array}
$$

(Here $\hat{E}$ is defined as $P+Q$ to match the usual notation used in the small pitch approximation.) 
For the synchronous relativistic case the deflecting force, or equivalently the deflecting field, is independent of the transverse coordinates and the longitudinal field components, which are shifted by $\pi / 2$ in the spatiotemporal phase, rise linearly with the distance to the axis.

Note, that for $\beta_{z}<1$ the transverse force is not constant over the transverse cross section of the structure, $F_{y}$ is not zero, and the longitudinal field components show a nonlinear dependence from the distance to the axis. As a result the slice emittance of a bunch will grow in both planes for low velocity beams.

\section{INFLUENCE OF THE LONGITUDINAL FIELD COMPONENTS ON THE PARTICLE DYNAMICS}

From Eq. (5) follows that the integrated transverse momentum transferred to a particle moving on a straight line (rigid beam approximation) through a region with an arbitrary electromagnetic field is related to the integrated longitudinal field by

$$
p_{\perp}=-i \frac{e}{k_{0} c} \int_{0}^{L} \nabla_{\perp} E_{z} d z .
$$

Equation (13) is referred to as Panofsky-Wenzel theorem. It is valid in this strict form only for synchronous motion with $v_{z}=v_{\mathrm{ph}}=c$. (Asynchronous field components average to zero when the integration length is long enough.) The rigid beam approximation excludes ponderomotive forces, however, for a force like derived in Eq. (12) ponderomotive forces are anyhow zero, because $F_{y}$ is everywhere zero and $F_{x}$ does not depend on $x$.

In accordance to Eq. (13) the transverse momentum $p_{\perp}=p_{x}$ and energy change of a bunch of particles passing through a deflecting structure of length $L$ following Eq. (12) read as:

$$
\begin{aligned}
p_{x} & =\frac{\mathrm{eV}}{c}[\sin (\varphi)+\cos (\varphi) \Delta \varphi] \\
E & =e k_{0} V x[\cos (\varphi)-\sin (\varphi) \Delta \varphi],
\end{aligned}
$$

where $V=\frac{\hat{E} L}{2}$ is the integrated deflecting voltage and a first order Taylor expansion of the phase $\varphi$ has been made. $\Delta \varphi$ denotes the position of a particle relative to the bunch center; $\Delta \varphi=-k_{0} \Delta z$.

While the first term in the momentum equation describes the average momentum gained by the bunch, the second term describes the spread due to the differences experienced by particles in the head and the tail of the bunch. In the fully deflecting mode, $\varphi=\pi / 2$, the momentum spread is to first order zero, while it is maximal at $\varphi=0$, the standard operation phase for cavity applications as diagnostics, crabbing, and emittance exchange.
Due to the dependence of the longitudinal field on the transverse coordinate the induced energy spread is on all phases uncorrelated:

$$
\sigma_{E}= \begin{cases}e k_{0} V \sigma_{x} & \text { for } \varphi=0 \\ e k_{0}^{2} V \sigma_{x} \sigma_{z} & \text { for } \varphi=\frac{\pi}{2}\end{cases}
$$

with the transverse rms beam size in the streaking direction $\sigma_{x}$ and the longitudinal rms bunch length $\sigma_{z}$. The PanofskyWenzel theorem, Eq. (13), as well as Eqs. (14) and (15) describe the beam dynamics to first order.

In second order the induced transverse momentum couples to the longitudinal magnetic field and the transverse particle position changes, which leads to an additional correlated energy spread of [4]

$$
\frac{\Delta E^{\mathrm{cor}}}{\Delta z}=\frac{\left(e k_{0} V\right)^{2}}{c p_{z}} \frac{L}{6},
$$

where $p_{z}$ is the longitudinal momentum of the particle.

The second order effects combine the cosinelike transverse momentum with the sinelike longitudinal field components and Eq. (16) is therefore valid for $\varphi=0$ and for $\varphi=\pi / 2$.

The momentum in the streaking direction leads in combination with the longitudinal magnetic field also to a force in direction perpendicular to the streaking plane:

$$
\hat{F}_{y}=-e \frac{p_{x}}{\gamma m_{0}} B_{z},
$$

with the rest mass of the particle $m_{0}$ and the relativistic Lorentz factor $\gamma$.

$B_{z}$ is expanded in a Taylor series as:

$$
B_{z}=-\frac{\hat{E}}{2 c} k_{0} y[\cos (\varphi)+\sin (\varphi) \Delta \varphi]
$$

and thus

$$
p_{y}=\frac{1}{c} \int \hat{F}_{y} d z=\frac{\left(e k_{0} V\right)^{2}}{2 c^{2} p_{z}} y \Delta z
$$

The transverse momentum $p_{y}$ is linear in the transverse position $y$, i.e., it is a focusing force, which depends however on the longitudinal position $\Delta z$ in the bunch. It leads thus to a projected emittance contribution of

$$
\varepsilon_{y}=\frac{\left(e k_{0} V\right)^{2}}{2 m_{0} c^{2} p_{z}} \sigma_{y}^{2} \sigma_{z}
$$

with the transverse rms beam size perpendicular to the streaking direction $\sigma_{y}$. Equations (19) and (20) are again valid for $\varphi=0$ and for $\varphi=\pi / 2$.

The emittance growth Eq. (20) in the direction perpendicular to the streaking force is a fundamental property of the deflecting field which does not depend on the design of the supporting structure or the operating mode of the cavity.

For present day beam and cavity parameters the emittance growth is very small, but it can not be eliminated. 
Additional transverse forces can still appear due the spatial harmonics especially in the end cells of rf structures and due to the backward traveling component in standing wave cavities. These forces average out in a first order approximation of the particle motion, but are relevant for second order effects $[20,21]$ and often dominate the beam dynamics in the plane perpendicular to the streaking direction.

\section{SUMMARY}

Transverse deflecting rf structures find nowadays numerous applications in particle accelerators. Regardless of the design and operating mode of the supporting structure, a synchronous transverse force, generated by the common interaction of the transverse electric and magnetic field components, is in general accompanied by both, electric as well as magnetic, longitudinal field components. The complete deflecting rf field has six field components and requires the representation by a linear combination of two basis vectors. The description in the HM-HE basis avoids convergence problems which are characteristic for the usual TM-TE basis for waves matched to the velocity of light.

Both, the longitudinal electric and the longitudinal magnetic field component lead to undesired and fundamental beam dynamics effects.

The longitudinal magnetic field, which has been ignored so far, in combination with the induced transverse momentum in the direction of the deflecting force, results in a small, but fundamental, emittance contribution in the direction perpendicular to the deflecting force.

\section{ACKNOWLEDGMENTS}

The author V.P. is supported by RFMEFI62117X0014 program of the Ministry of Science and Education.

[1] L. Bellantoni, H. T. Edwards, M. McAshan et al., Design and measurement of a deflecting mode cavity for an rf separator, in Proceedings of the 2001 Particle Accelerator Conference, Chicago, IL (IEEE, New York, 2001), http://accelconf.web .cern.ch/AccelConf/p01/PAPERS/MPPH129.PDF.

[2] D. Alesini, F. Marcellini, A. Ghigo et al., The new rf deflectors for the CTF3 combiner ring, in Proceedings of the 23rd Particle Accelerator Conference, Vancouver, Canada, 2009 (IEEE, Piscataway, NJ, 2009), http:// accelconf.web.cern.ch/AccelConf/PAC2009/papers/we1 pbc04.pdf.

[3] D. Ratner, C. Behrens, Y. Ding, Z. Huang, A. Marinelli, T. Maxwell, and F. Zhou, Time-resolved imaging of the microbunching instability and energy spread at the Linac Coherent Light Source, Phys. Rev. Accel. Beams 18, 030704, 2015.

[4] P. Emma and M. Cornacchia, Transverse to longitudinal emittance exchange, Phys. Rev. Accel. Beams 5, 084001 (2002).
[5] D. R. Brett, R. B. Appleby, R. De Maria, J. Barranco Garcia, R. Tomás Garcia, B. Hall, and G. Burt, Accurate crab cavity modeling for the high luminosity Large Hadron Collider, Phys. Rev. Accel. Beams 17, 104001 (2014).

[6] W. K. H. Panofsky and W. A. Wenzel, Some considerations concerning the transverse deflection of charged particles in radio-frequency fields, Rev. Sci. Instrum. 27, 967 (1956).

[7] P. B. Wilson, Introduction to wake fields and wake potentials, AIP Conf. Proc. 184, 525 (1989).

[8] O. Lambertson, Dynamic devices-pickups and kickers, in Physics of Accelerators, AIP Conf. Proc. 153, 1413 (1987).

[9] Y. Garault, Etude d'une classe d'ondes electromagnetiques guideres: Les ondes EH application aux structures deflectrices pour les separateurs a obde progressive de particules relativistes, CERN Report No. CERN 64-43, 1964.

[10] K. Floettmann and V. Paramonov, Beam dynamics in transverse deflecting rf structures, Phys. Rev. Accel. Beams 17, 024001 (2014).

[11] V. V. Paramonov, Field distribution analysis in deflecting structures, Report No. DESY 13-018, 2013 [arXiv:1302 $.5306 v 1]$.

[12] M. Ivanyan, A. Grigoryan, A. Tsakanian, and V. Tsakanov, Narrow-band impedance of a round metallic pipe with a low conductive thin layer, Phys. Rev. Accel. Beams 17, 021302, 2014.

[13] A. Novokhatsky, M. Timm, and T. Weiland, The surface roughness wake field effect, in Proceedings of the 1998 International Computational Accelerator Physics Conference, Monterey, 1998, http://www.slac.stanford.edu/ xorg/icap98/.

[14] V. A. Vagin and V. I. Kotov, Investigation of hybrid waves in a circular waveguide partially filled with dielectric, J. Tech. Phys. 35, 1273 (1965) [Soviet Physics-Technical Physics 10, 987 (1966)].

[15] Ch. T. M. Chang and J. W. Dawson, Propagation of electromagnetic waves in a partially dielectric filled circular waveguide, J. Appl. Phys. 41, 4493 (1970).

[16] F. Lemery, K. Floettmann, T. Vinatier et al., A transverse deflection structure with dielectric-lined waveguide in the sub-THz regime, Proceedings of IPAC 2017, Copenhagen, 2017, http://accelconf.web.cern.ch/AccelConf/ipac2017/ papers/mopab052.pdf.

[17] F. Ahr et al., Narrowband terahertz generation with chirped-and-delayed laser pulses in periodically poled lithium niobate, Opt. Lett. 42, 2118, 2017.

[18] H. Hahn, Deflecting mode in circular iris-loaded waveguides, Rev. Sci. Instrum. 34, 1094 (1963).

[19] V. Paramonov and K. Floettmann, Fundamental characteristics of transverse deflecting fields, Report No. DESY 18103, 2018 [arXiv:1806.11023].

[20] S. Reiche, J. B. Rosenzweig, S. Anderson, P. Frigola, M. Hogan, A. Murokh, C. Pellegrini, L. Serafini, G. Travish, and A. Tremaine, Experimental confirmation of transverse focusing and adiabatic damping in a standing wave linear accelerator, Phys. Rev. E 56, 3572 (1997).

[21] A. Opanasenko, Applicability of the Panofsky-Wenzel theorem, in Proceedings of the International Particle Accelerator Conference, Kyoto, Japan (ICR, Kyoto, 2010), http://accelconf.web.cern.ch/AccelConf/IPAC10/ papers/thpe085.pdf. 